«Системні технології» 5 (130) 2020 «System technologies»

DOI 10.34185/1562-9945-5-130-2020-10

УДК 004.02

C.M. Вовк

\title{
ПРИМЕНЕНИЕ ФУНКЦИОНАЛА КВАЗИПРОТЯЖЕННОСТИ В ЗАДАЧАХ АППРОКСИМАЦИИ ИСКАЖЕННЫХ ДАННЫХ
}

Аннотация. Исследована эффективность применения функционала квазипротяженности для решения задач аппроксимации данных, описываемых параметрической моделью. Представлены результаты теоретического и численного анализа, которые демонстрируют особенности поведения этого функционала как целевой функции линейного и нелинейного параметров модели данных для разных уровней шума в предположении, что параметры модели принимают одно или несколько значений. Сформулированы рекомендации по эффективному использованию функционала квазипротяженности для решения задач аппроксимации данных.

Ключевые слова: функционал, аппроксимация, данные.

Введение. Функционал квазипротяженности предназначен для решения задач обработки данных в условиях, когда исходные данные искажены шумом и аномальными значениями [1]. Для решения задач аппроксимации искаженных данных посредством заданной параметрической модели этот функционал целесообразно строить на основе функции невязки между данными и их моделью [2]. В данной работе представлены результаты исследования эффективности применения функционала квазипротяженности невязки в задачах аппроксимации искаженных данных, которые включают результаты теоретического и численного анализа поведения функционала квазипротяженности для случаев, когда на интервале наблюдения данных неизвестные параметры принимают одно или несколько значений, а данные искажены аддитивным шумом.

Постановка задачи и цель исследований. Постановка задачи заключается в исследовании эффективности применения функционала квазипротяженности, построенного на основе функции невязки между данными и их параметрической моделью, для решения задач аппрокси-

(C) Вовк C.M., 2020 


\section{«Системні технології» 5 (130) 2020 «System technologies»}

мации искаженных данных. Целью работы является формирование рекомендаций по эффективному использованию функционала квазипротяженности для решения задач аппроксимации искаженных данных.

Анализ последних исследований и публикаций. Согласно [1], постановка задачи аппроксимации данных заданной параметрической моделью заключается в минимизации функционала квазипротяженности функции невязки между этими данными и их моделью. Для непрерывного случая этот функционал задается в виде:

$$
E^{(\alpha, \beta, q)}[r(x)]=\int_{-\infty}^{\infty} \psi_{S}^{(\alpha, \beta, q)}[r(x)] d x,
$$

где $r(x)$ есть функция невязки, $\psi_{S}^{(\alpha, \beta, q)}(x)=k_{S}^{(\alpha, \beta, q)}\left[\left(1+|x / \alpha|^{q}\right)^{\beta / q}-1\right]$ есть стоимостная функция, $\alpha$ - параметр сглаживания; $q$ - параметр степени сглаживания, причем $0<q<\infty ; \beta$ - параметр формы, причем $-\infty<\beta \leq 1$ и $\beta<q ; k_{S}^{(\alpha, \beta, q)}=1 /\left[\left(1+\left|x_{0} / \alpha\right|^{q}\right)^{\beta / q}-1\right] ; x_{0}-$ точка нормировки $\psi_{S}^{(\alpha, \beta, q)}(x)$ на единицу. Параметры $\alpha, \beta, q$ имеют смысл свободных параметров и позволяют менять поведение стоимостной функции, обеспечивая широкие возможности по настройке процесса обработки данных на текущую шумовую обстановку [3]. В [4] отмечено, что функционал (1) не является выпуклым за исключением своих крайних случаев, а его первая вариация в точке минимума может не существовать. Однако в отличие от традиционно используемого в задачах аппроксимации квадратичного функционала [5], функционал квазипротяженности (1) предоставляет возможность решения задач аппроксимации в условиях, когда исходные данные искажены грубыми ошибками, а неизвестные параметры могут принимать не одно, а несколько значений [4]. Подобными свойствами также обладают функционалы, построенные на основе квазинормы пространства $L_{p} ; 0<p<1$ [6] или на основе "минимаксно-вогнутой штрафной функции" (minimax-concave penalty function) в случае их применения к невязке решения [7].

Основная часть. Эффективность применения различных функционалов в задачах аппроксимации данных определяется, в первую очередь, наличием у них локальных минимумов, отвечающих искомым зна- 
«Системні технології» 5 (130) 2020 «System technologies»

чениям неизвестных параметров модели. Для непрерывного случая из (1) можно получить, что функционал квазипротяженности для случая неизвестного линейного параметра $A$ принимает вид целевой функции:

$$
E^{(\alpha, \beta, q)}(A)=k_{S}^{(\alpha, \beta, q)} \int_{X}\left[\left(1+|(g(x)-A f(x)) / \alpha|^{q}\right)^{\beta / q}-1\right] d x,
$$

где функция $g(x)$ описывает данные, $f(x)$ - известную форму модели данных, а $X$ - область наблюдения. В (2) для $0<\beta<1$ имеем $k_{S}^{(\alpha, \beta, q)}>0$, для $-\infty<\beta<0$ имеем $k_{S}^{(\alpha, \beta, q)}<0$, а для $\beta \rightarrow \pm 0$ вместо (2) получаем:

$$
E^{(\alpha, 0, q)}(A)=k_{S}^{(\alpha, 0, q)} \int_{X} \ln \left[1+|(g(x)-A f(x)) / \alpha|^{q}\right] d x,
$$

где $k_{S}^{(\alpha, 0, q)}=1 / \ln \left[1+\left|x_{0} / \alpha\right|^{q}\right]>0$. Из (2) и (3) видно, что для тех значений параметра $A$, которые обращают в нуль модуль невязки $|g(x)-A f(x)|$, на графике целевой функции могут возникать локальные минимумы, отвечающие этим значениям параметра $A$.

Аналогично (2)-(3), функционал квазипротяженности для случая неизвестного нелинейного параметра $\theta$ имеет вид целевой функции:

$$
\begin{gathered}
E^{(\alpha, \beta, q)}(\theta)=k_{S}^{(\alpha, \beta, q)} \int_{X}\left[\left(1+|(g(x)-s(x ; \theta)) / \alpha|^{q}\right)^{\beta / q}-1\right] d x ; \beta \neq 0, \\
E^{(\alpha, 0, q)}(\theta)=k_{S}^{(\alpha, 0, q)} \int_{X} \ln \left[1+|(g(x)-s(x ; \theta)) / \alpha|^{q}\right] d x ; \quad \beta=0,
\end{gathered}
$$

где функция $s(x ; \theta)$ задает модель данных с точностью до неизвестного параметра $\theta$. Из (4)-(5) видно, что для тех значений параметра $\theta$, которые обращают в нуль модуль невязки $|g(x)-s(x ; \theta)|$, на графике целевой функции также могут возникать локальные минимумы, отвечающие этим значениям $\theta$. В силу нелинейности параметра $\theta$, эти локальные минимумы могут возникать даже тогда, когда при тех же условиях целевая функция для линейного параметра является выпуклой.

Рассмотрим поведение функционала квазипротяженности при наличии шума в данных для частного случая $\alpha=0$ и $0<\beta<1$, когда этот функционал отражает понятие квазинормы ненормированного пространства $L_{\beta} ; 0<\beta<1$. В этом случае возможен следующий теоретический анализ искажений истинного значения линейного параметра модели. Пусть шум $n(x)$ проявляется в том, что для каждого $x \in X$ вместо 
«Системні технології» 5 (130) 2020 «System technologies»

$g(x)=A_{\text {true }} f(x)$, где $A_{\text {true }}$ есть истинное значение $A$, фиксируются значения $g(x)=\left(A_{\text {true }}+\xi\right) f(x)$, т.е. $n(x)=\xi f(x)$, где $\xi$ есть случайная величина, распределенная по закону $p(\xi)$ и для которой существует математическое ожидание. Тогда невязка между $g(x)=\left(A_{t r u e}+\xi\right) f(x)$ и моделью $A f(x) \quad$ в каждой точке $\quad x \in X \quad$ равна: $r(x)=\left(A_{\text {true }}+\xi\right) f(x)-A f(x)=\left(A_{\text {true }}-A+\xi\right) f(x)$ и вместо (2) имеем:

$$
E^{(0, \beta, q)}(A ; \xi)=c(\beta) \cdot\left|\left(A-A_{\text {true }}\right)-\xi\right|^{\beta} \text {, }
$$

где $c(\beta)=\int_{x}|f(x)|^{\beta} d x /\left|x_{0}\right|^{\beta} ; 0<\beta \leq 1$. Усредняя по $\xi$, из (6) получаем:

$$
\mathbf{M}\left[E^{(0, \beta, q)}(A ; \xi)\right]=c(\beta) \cdot \int_{-\infty}^{\infty}\left|\left(A-A_{\text {true }}\right)-\xi\right|^{\beta} p(\xi) d \xi .
$$

Соотношение (7) указывает, что в этом случае имеем свертку функции (функционала) $E^{(\beta)}(A)=c(\beta) \cdot\left|A-A_{\text {true }}\right|^{\beta}$ и закона распределения $p(\xi)$. Очевидно, что эта свертка приводит к сглаживанию функционала $E^{(\beta)}(A)$, вызывая уменьшение глубины его минимумов и их уширение. Однако детали такого сглаживания могут быть разными. Так, если закон распределения $p(\xi)$ имеет «гладкую вершину» (например, как закон Гаусса), то локальные минимумы функционала $E^{(\beta)}(A)$ будут сглажены и закруглены. Однако для «островершинного» закона распределения (например, для закона Лапласа) сглаживание не будет закруглять локальные минимумы. При этом для дискретного случая можно отметить, что в предположении эргодичности случайного процесса такое сглаживание будет описываться соотношением (7) тем лучше, чем будет большим число дискретных точек. В дополнение отметим, что при отсутствии шума имеем: $p(\xi)=\delta(\xi)$, где $\delta(\xi)$ есть дельта-функция Дирака, и тогда из (7) получаем $E^{(\beta)}(A)$.

В общем случае исследование поведения функционала квазипротяженности целесообразно выполнять посредством численного моделирования. Это обусловлено, в первую очередь, сложностью необходимых аналитических преобразований, а также быстротой численного анализа самых различных ситуаций, связанных с шумом. 
«Системні технології» 5 (130) 2020 «System technologies»

На рис.1 приведен пример численного моделирования функционала квазипротяженности $E^{(\beta)}(A) ; \beta=1 / 2$ для случая линейного параметра, которым являлась амплитуда модельной функции, которая принимала одно ( $A=1$; рис. 1а) и два ( $A=1$ и $A=2$; рис. 1б) значения. Исходные данные искажались гауссовским шумом $\mathbf{N}\left(0, \sigma^{2}\right)$. Видно, что полученные результаты подтверждают результаты теоретического анализа, согласно которым сглаживание локальных минимумов становится тем большим, чем большим становится шум.
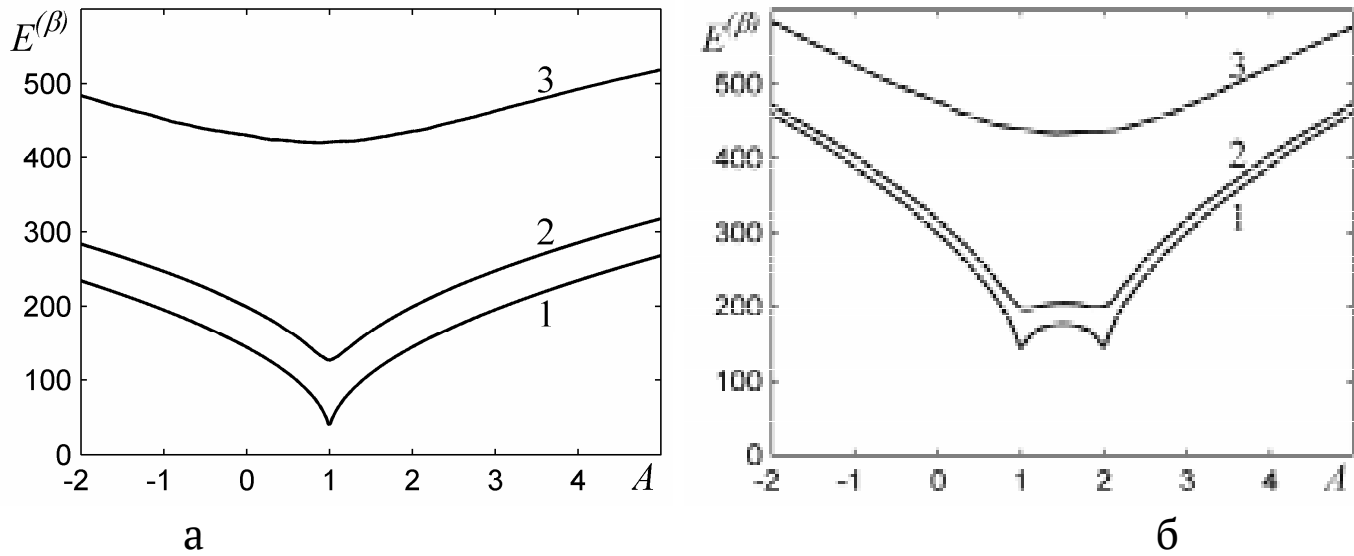

Рисунок 1 - Целевая функция функционала квазипротяженности для линейного параметра, который принимает одно (а) и два значения (б); кривые 1, 2 и 3 получены для $\sigma=0.01, \sigma=0.1$ и $\sigma=1$, соответственно

На рис.2 приведен пример численного моделирования функционала $E^{(\beta)}(A) ; \beta=1 / 2$ для нелинейного параметра, которым являлась частота синусоиды единичной амплитуды, принимающая одно $(v=1$; рис. 2a) и два ( $v=1$ и $v=2$; рис. 2б) значения. Исходные данные искажались гауссовским шумом $\mathbf{N}\left(0, \sigma^{2}\right)$. Видно, что малый уровень шума практически не влияет на поведение функционала (кривые 1 и 2), тогда как большой шум (кривая 3) сильно заглаживает его локальные минимумы, делая их трудно различимыми на осциллирующем фоне. 
«Системні технології» 5 (130) 2020 «System technologies»
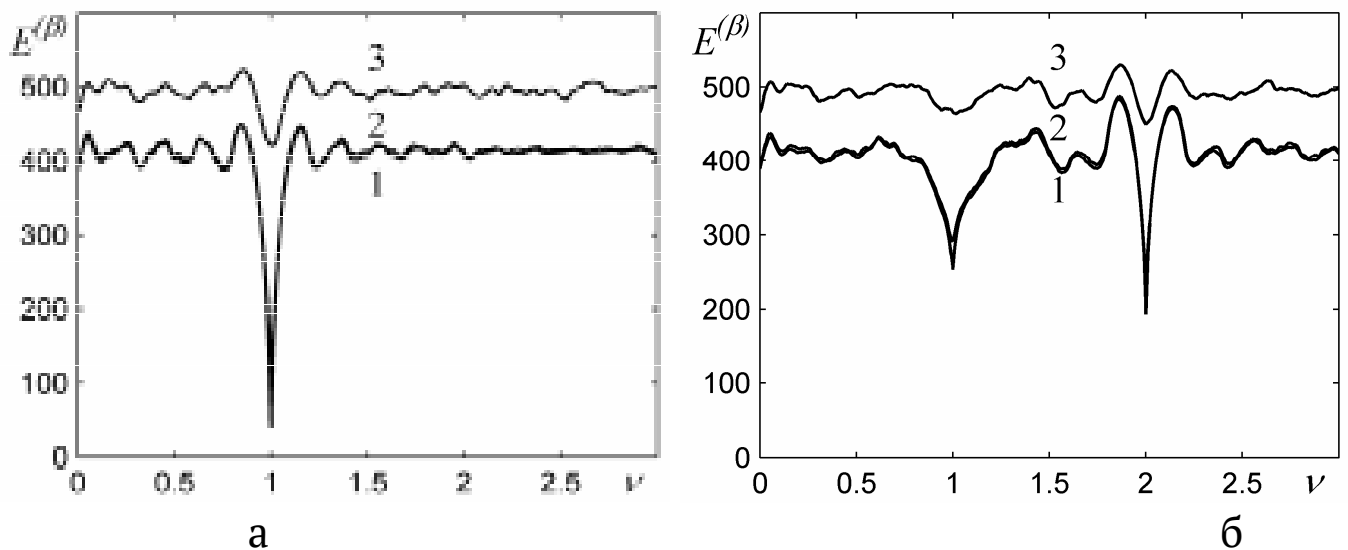

Рисунок 2 - Целевая функция функционала квазипротяженности для нелинейного параметра, который принимает одно (а) и два значения (б); кривые 1, 2 и 3 получены для $\sigma=0.01, \sigma=0.1$ и $\sigma=1$, соответственно

Численное моделирование для случая $\alpha>0$ показало, что функционал $E^{(\alpha, \beta, q)}$ является эффективным тогда, когда отношение "сигнал/шум" больше 10 и $\alpha \approx \sigma$. В частности, последнее справедливо для шума с законом распределения Коши, когда $\alpha=\sigma, \beta=0, q=2$.

Основываясь на результатах данных исследований, можно сформулировать такие рекомендации по использованию функционала квазипротяженности для эффективного решения задач аппроксимации: 1) необходим выбор адекватной параметрической модели искаженных данных; 2) необходима настройка функционала квазипротяженности на текущую шумовую обстановку; 3) необходимо применение методов оптимизации неунимодальных функций; 4) желательно комбинирование функционала квазипротяженности невязки с теми ограничениями, которые позволяют привлечь дополнительную априорную информацию.

Выводы. Эффективность применения функционала квазипротяженности в задачах аппроксимации данных обусловлена как возможностью настройки процесса аппроксимации на текущую шумовую обстановку, так и возможностью аппроксимации искаженных данных в условиях, когда неизвестные параметры модели принимают несколько значений. Увеличение уровня шума в данных приводит к ухудшению качества аппроксимации. 


\section{«Системні технології» 5 (130) 2020 «System technologies»}

\section{ЛИТЕРАТУРА / ЛІТЕРАТУРА}

1. Вовк С. М. Постановка задач обработки данных на основе критерия минимума протяженности / С. М. Вовк // Радіоелектроніка, інформатика, управління. - 2019. - N.1 -C. 157-166. DOI: 10.15588/1607-3274-2019-1-15.

2. Вовк С. М. Критерій мінімуму протяжності / С. М. Вовк // Системні технології. Регіональний міжвузівський збірник наукових праць. Випуск 1 (120). - Дніпро, 2019. - С. 19 - 25.

3. Вовк С. М. Метод обробки даних в умовах складного шумового оточення / С. М. Вовк, В. В. Гнатушенко // Науковий вісник Таврійського державного агротехнологічного університету. - 2018. - Вип. 8. - Т.2. - С. 19. DOI: $10.31388 / 2220-8674-2018-2$.

4. Вовк С.М. Оценивание параметра с несколькими значениями / С. М. Вовк, О. Н. Прокопчук // Радіоелектроніка, інформатика, управління. 2019. - N. 4. - C. 14-24. DOI: 10.15588/1607-3274-2019-4-2

5. Wolberg J. Data Analysis Using the Method of Least Squares: Extracting the Most Information from Experiments / J. Wolberg. - Berlin: SpringerVerlag, 2005. - $250 \mathrm{p}$.

6. Ming-Jun Lai and Jingyue Wang An Unconstrained $\ell$ q Minimization with $0<$ $\mathrm{q} \leqslant 1$ for Sparse Solution of Under-determined Linear Systems // SIAM Journal on Optimization, 2011. - V.21. - N 1. - P. 82-101.

7. Liu Q. Robust Sparse Recovery via Weakly Convex Optimization in Impulsive Noise / Q. Liu, C. Yang, Y. Gu, H. C. So // Signal Processing. - 2018. - Vol. 152. - P. 84-89. DOI: =https://doi.org/10.1016/j.sigpro.2018.05.020.

\section{REFERENCES}

1. Vovk S. M. Postanovka zadach obrabotki dannyih na osnove kriteriya minimuma protyazhennosti / S. M. Vovk // Radioelektronika, informatyka, upravlinnia. - 2019. - N.1 - S. 157-166. DOI: 10.15588/1607-3274-2019-1-15.

2. Vovk S. M. Kryterii minimumu protiazhnosti / S. M. Vovk // Systemni tekhnolohii. Rehionalnyi mizhvuzivskyi zbirnyk naukovykh prats. Vypusk 1 (120). - Dnipro, 2019. - C. 19 - 25.

3. Vovk S. M. Metod obrobky danykh v umovakh skladnoho shumovoho otochennia / S. M. Vovk, V. V. Hnatushenko // Naukovyi visnyk Tavriiskoho derz- 
«Системні технології» 5 (130) 2020 «System technologies»

havnoho ahrotekhnolohichnoho universytetu. - 2018. - Vyp. 8. - T.2. - S. 19. DOI: 10.31388/2220-8674-2018-2.

4. Vovk S.M. Otsenivanie parametra s neskolkimi znacheniyami / S. M. Vovk, O. N. Prokopchuk // Radioelektronika, informatyka, upravlinnia. - 2019. - N. 4. - S. 14-24. DOI: 10.15588/1607-3274-2019-4-2

5. Wolberg J. Data Analysis Using the Method of Least Squares: Extracting the Most Information from Experiments / J. Wolberg. - Berlin: SpringerVerlag, 2005. $-250 \mathrm{p}$.

6. Ming-Jun Lai and Jingyue Wang An Unconstrained $\ell q$ Minimization with $0<$ $\mathrm{q} \leqslant 1$ for Sparse Solution of Under-determined Linear Systems // SIAM Journal on Optimization, 2011. - V.21. - N 1. - P. 82-101.

7. Liu Q. Robust Sparse Recovery via Weakly Convex Optimization in Impulsive Noise / Q. Liu, C. Yang, Y. Gu, H. C. So // Signal Processing. - 2018. - Vol. 152. - P. 84-89. DOI: =https://doi.org/10.1016/j.sigpro.2018.05.020.

Застосування функціонала квазіпротяжності

Received 17.03.2020. Accepted 23.03.2020.

в задачах апроксимації спотворених даних

Досліджена ефективність застосування функціонала квазіпротяжності для розв'язання задач апроксимації даних, описуваних параметричною моделлю. Представлені результати теоретичного й чисельного аналізу, які демонструють особливості поведінки цього функціонала як цільової функції лінійного й нелінійного параметрів моделі даних для різних рівнів шуму в припущенні, що параметри моделі приймають одне або кілька значень. Сформульовані рекомендації з ефективного використання функціонала квазіпротяжності для розв'язання задач апроксимації даних.

\section{Using of functional of the quasi-extent}

in the problems of approximation of distorted data

The quasi-extent functional is intended to process the data distorted by noise and anomalous values. To solve the approximation problem of distorted data described by a parametric model it is advisable to build this functional on the basis of a residual function. The goal of this paper is to formulate the recommendations to effective use of the quasi-extent functional for approximation of distorted data.

The effectiveness of using the various functionals in the data approximation problem is determined, first of all, by the presence of such local minima that correspond to the true values of unknown model parameters closely. In this paper, it is noted that the quasi-extent functional of solution residual contains such minima both for the case of a linear parameter of data model and for the case of a non-linear parameter of data model. In particular, by setting the corresponding values of free parameters, the quasi-extent functional can be tuned to a quasinorm of the space $L_{p} ; 0<p<1$. While the noise is absence, the local minima of this quasi-extent functional will exactly correspond to those values of unknown data model parameter, when using 86 


\section{«Системні технологіï» 5 (130) 2020 «System technologies»}

which the residual function strictly equals to zero on a certain argument interval, i.e. when at this interval the model coincides with the data exactly. However, in the presence of moderate noise, such minima are smoothed out, leading to a decrease in their depth and broadening. Moreover, in the presence of high noise, the neighboring local minima that correspond to the different values of the sought parameter can either merge into one minimum (for the case of linear parameter), or become indistinguishable against the general background of values (for the case of nonlinear parameter). Taking the features of the quasi-extent functional into account, the recommendations about its effective use to solve the data approximation problem are formulated. The effectiveness of using the quasi-extent functional to solve the data approximation problem is due to the possibility of tuning of approximation process to the current noise environment and the possibility of approximating of distorted data under condition when unknown model parameters take several values.

Вовк Сергей Михайлович - к.ф.-м.н., доцент, Днепровский национальный университет, доцент кафедры компьютерных наук и информационных технологий.

Вовк Сергій Михайлович - к.ф.-м.н., доцент, Дніпровський національний університет, доцент кафедри комп'ютерних наук та інформаційних технологій.

Vovk Serhii - Ph.D, Associate Professor, Oles Honchar Dnipro National University, Associate Professor of Department of Computer Science and Information Technology. 